Number of patients exposed to the discontinuation of first TNF- $\alpha$ blocker

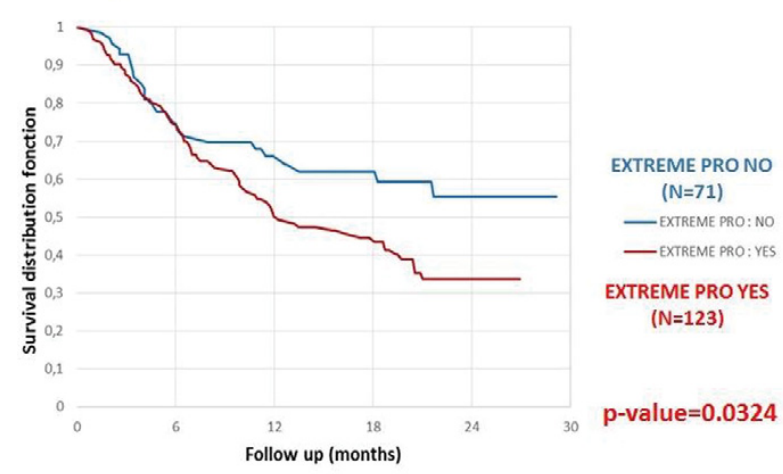

Figure 1. Impact of extreme PRO on first TNF- $\alpha$ blocker retention rate (first 2years) [Kaplan Meier curves and Log Rank test]

Acknowledgements: The DESIR cohort is financially supported by unrestricted grants from the French Society of Rheumatology and Pfizer France.

Disclosure of Interest: B. Meghnathi Grant/research support from: ASAS Society (ASAS FELLOW), A. Etcheto: None declared, P. Claudepierre: None declared, M. Dougados: None declared, A. Moltó: None declared

DOI: 10.1136/annrheumdis-2017-eular.3827

\section{SAT0387 "DO NOT DO" RECOMMENDATIONS IN THE MANAGEMENT OF COMORBIDITY IN PATIENTS WITH AXIAL SPONDYLOARTHRITIS. GECOAX PROJECT}

J.C. Torre-Alonso ${ }^{1}$, C. González ${ }^{2}$, S. Castañeda ${ }^{3}$, R. Curbelo ${ }^{4}$, E. Collantes ${ }^{5}$, M.V. Hernández ${ }^{6}$, A. Urruticoechea-Arana ${ }^{7}$, J.C. Nieto ${ }^{2}$, J. García ${ }^{8}$,

M.Á. Abad ${ }^{9}$, J. Ramírez ${ }^{6}$, C. Suárez ${ }^{10}$, R. Dalmau ${ }^{11}$, M.D. Martín-Arranz ${ }^{12}$, L. León ${ }^{13}$, J.C. Hermosa ${ }^{14}$, J.C. Obaya ${ }^{15}$, T. Otón ${ }^{4}$, L. Carmona ${ }^{4}$

${ }^{1}$ Rheumatology, HU Monte Naranco, Oviedo; ${ }^{2}$ Rheumatology, HU Gregorio

Marañón; ${ }^{3}$ Rheumatology, HU la Princesa, IIS-IP; ${ }^{4}$ InMusc, Madrid;

${ }^{5}$ Rheumatology, HU Reina Sofía, Córdoba: ${ }^{6}$ Rheumatology, HU Clinic $i$

Provincial, Barcelona; ${ }^{7}$ Rheumatology, $\mathrm{H}$ Can Misses, Ibiza; ${ }^{8}$ Rheumatology, HU 12 de Octubre, Madrid; ${ }^{9}$ Rheumatology, H de Plasencia, Plasencia; ${ }^{10}$ Internal Medicine, HU la Princesa, IIS-IP; ${ }^{11}$ Cardiology; ${ }^{2}$ Gastroenterology, HU la Paz;

${ }^{13}$ Psychology, UCJC, Madrid; ${ }^{14}$ Centro de Salud Ciudades, Getafe; ${ }^{15}$ Centro de Salud Alcobendas, Alcobendas, Spain

Background: During the development of recommendations and implementation aids of the GECOAx project, the importance of avoiding certain situations was highlighted.

Objectives: To recognize what prescriptions, risk assessments, or preventive strategies are wrong practices and should thus be avoided in clinical practice. To establish not to do recommendations in the management of the comorbidity of AxSpA.

Methods: A multidisciplinary group was selected [10 rheumatologists, 1 internist, 1 cardiologist, 1 gastroenterologist, 1 psychologist and 2 family physicians]. With the support of 3 methodologists, and after interactions aimed to edit a document for the management of comorbidity launched by the same panel, a list of Not to do recommendations was issued. In a discussion meeting, evidence was provided to support the recommendations, items without sufficient basis were removed, and the final list was produced.

Results: A summary list of Not to do recommendations (Table 1) was issued.

Table 1

- DO NOT prescribe NSAIDs to patients with high cardiovascular (CV) risk and particularly with

hypertension.

- DO NOT prescribe NSAIDs to patients with CKD, heart failure or liver cirrhosis and, if

necessary, exert caution.

- DO NOT use CV risk scores in patients who already suffered a CV event or those with multiple risk factors (smoking, obesity, sedentary lifestyle, DM, hypertension, dyslipidemia) or a family history of premature $\mathrm{CV}$ disease; All should be considered high CV risk.

- DO NOT base renal disease screening on a single glomerular filtration test and/or albuminuria (ALWAYS should be confirmed); serum creatinine should not be used as the only test to evaluate renal function.

- DO NOT administer biological therapy in case of active, serious and uncontrolled infection, sepsis or risk of sepsis or tuberculosis or without a previous screening of chronic HBV, HCV, HIV and TB.

- DO NOT repeat HBV vaccination unless HBV antibody levels are not achieved.

DO NOT vaccinate a patient in therapy with biological agents or in immunosuppressive

treatment with live viruses

Conclusions: These recommendations aim to avoid making common mistakes in clinical practice and to help better management of frequent comorbidity in patients with $\mathrm{AxSpA}$.

Acknowledgements: This project was funded by Merck Sharp \& Dohme Spain. Merck Sharp \& Dohme had no influence on either the development of the project or the final content of the manuscript.
Disclosure of Interest: None declared

DOI: 10.1136/annrheumdis-2017-eular.5029

\section{SAT0388 ANALYSIS OF THE MUSCULOSKELETAL MANIFESTATIONS IN INFLAMMATORY BOWEL DISEASE PATIENTS AND ITS} RELATIONSHIP WITH BIOLOGICAL TREATMENT

S. Mainero Mendoza ${ }^{1,2}$, C.M. Feced Olmos ${ }^{1}$, L. Gonzalez Puig ${ }^{1}$, J. Ivorra Cortes $^{1}$, I. Martínez Cordellat ${ }^{1}$, C. Nájera Herranz ${ }^{1}$, R. Negueroles Albuixech ${ }^{1}$ J.E. Oller Rodriguez ${ }^{1}$, F.M. Ortiz-Sanjuan ${ }^{1}$, E. Vicens Bernabeu ${ }^{1}$, E. Grau Garcia $^{1}$, E. Labrador Sánchez ${ }^{1}$, K. Arévalo Ruales ${ }^{1}$, J.J. Fragio Gil ${ }^{1}$, R. González Mazarío ${ }^{1}$, C. Alcañiz Escandell ${ }^{1}$, I. Cánovas Olmos ${ }^{1}$, I. Chalmeta Verdejo ${ }^{1}$, V. Fornés Ferrer ${ }^{3}$, J.A. Román Ivorra ${ }^{1,2} .{ }^{1}$ Rheumatology Department, HUP la Fe; ${ }^{2}$ Medical School, UCV; ${ }^{3}$ Biostatistics Unit, IIS la Fe, Valencia, Spain

Background: Crohn's disease (CD) and ulcerative colitis (UC) are the main entities of inflammatory bowel disease (IBD). Both present extraintestinal manifestations that do not always depend on the IBD activity. The most common manifestations involve the musculoskeletal system and they are included in the seronegative spondiloarthritis group. If there is active or known IBD, treatment of this is priority because it usually improves joint disease. However, joint disease can also have an independent course of the intestinal manifestations as in patients with IBD and ankylosing spondylitis (AS).

Objectives: To analyze the prevalence of extraintestinal manifestations in IBD patients and treatment provided.

Methods: Retrospective observational analysis of IBD patients that have been remitted to the rheumatology department of HUP La Fe with musculoskeletal manifestations. Demographic, clinical and treatment data of patient were collected Biostatistical analysis with $\mathrm{R}$ (3.3.2.) was performed.

Results: We recruited 183 patients diagnosed with IBD (57.4\% women), 117 with CD and 66 with UC, with a mean age at diagnosis of $37.03 \pm 14.02$ years old. 29 of them have axial affection and 51 peripheral affection, and simultaneously in 22 cases. We observed no statistical differences in axial or peripheral affection according to the IBD diagnosis. 79 cases were on biological therapy, and these treatments were conducted by Rheumatology in the $44 \%$ of cases and by Digestive Department in the $66 \%$ of cases. We observed that patients with axial affection present higher probability that the treatment has been conducted by Rheumatology ( $\mathrm{P}=0.007)$, and broken down axial affections AS diagnosis had the most probability to be conducted by Rheumatology ( $n=36 P=0.0102)$. Related to peripheral manifestations, uveitis diagnosis had the most probability to be conducted by Rheumatology $(n=14 P=0.0337$ ).

Conclusions: In our patient series with IBD and musculoskeletal manifestations, the most common were peripheral affection. Among patients with IBD and axial and/or peripheral manifestation, $44 \%$ were conducted by Rheumatology, and are cases with axial predominance, where IBD treatment does not improve musculoskeletal disease and a primary spondyloarthritis treatment is needed.

Disclosure of Interest: None declared

DOI: 10.1136/annrheumdis-2017-eular.5687

\section{SAT0389 COMPARISON OF ANKYLOSING SPONDYLITIS AND NON-RADIOGRAPHIC AXIAL SPONDYLOARTHRITIS IN A MULTI-ETHNIC ASIAN POPULATION OF SINGAPORE}

C. Hong ${ }^{1}$, Y.H. Kwan ${ }^{2}$, W. Fong ${ }^{1} .{ }^{1}$ Department of Rheumatology and Immunology, Singapore General Hospital; ${ }^{2}$ Programme in Health Services and Systems Research, Duke-NUS Medical School, Singapore, Singapore

Background: The relationship between non-radiographic axial spondyloarthritis (nr-axSpA) and ankylosing spondylitis (AS) is currently debated. Till date, there is no study exploring the differences between AS and nr-axSpA in Asia.

Objectives: The primary objective of this study was to compare clinical characteristics, disease activity, patient-reported outcomes and associated comorbidities between patients with $\mathrm{AS}$ and nr-axSpA in a multiethnic Asian population of Singapore.

Methods: All patients fulfilled 2009 ASAS classification criteria for axial SpA. Of these, all AS patients fulfilled the modified New York criteria. AS and nr-axSpA patients were retrieved from the PREcision medicine in SPONdyloarthritis for Better Outcomes and Disease Remission (PRESPOND) registry in Singapore General Hospital. Patients were followed up over 2 years. Baseline characteristics, medications, disease activity, patient-reported outcomes and inflammatory markers prior and 6 months post treatment were recorded using standardized questionnaires.

Results: 262 AxSpA patients (82\% Chinese, $79 \%$ males) were included. Current mean age (S.D.) was 41.7 (13.7) years, mean age of diagnosis was 31.7 (12.5) years, mean length of disease was 10.1 (8.3) years and body mass index was 24.7 (6.3) $\mathrm{kg} / \mathrm{m}^{2}$, which was similar between AS and $\mathrm{nr}$-axSpA patients. AS patients were older [mean age 42.7 (13.5) vs 37.4 (13.8) years, $p=0.02$ ], had longer disease duration [mean disease duration 10.9 (8.7) vs 6.4 (4.8) years, $\mathrm{p}<0.01$ ], more frequently HLA-B27 positive ( $82 \%$ vs $68 \%, p=0.03)$, associated with uveitis ( $33 \%$ vs $17 \%, p=0.03)$, and hypertensive $(17 \%$ vs $0 \%, p<0.01)$ compared to $\mathrm{nr}-\mathrm{AxSpA}$ respectively. $\mathrm{nr}$-axSpA patients had higher BASDAI [mean BASDAI 4.2 (1.6) vs 3.5 (1.9), $\mathrm{p}=0.02$ ], BAS-G [mean BAS-G 4.7 (1.7) vs 3.9 (2.1), $p<0.01$ ] and ASQOL [mean ASQOL 4.9 (4.8) vs $3.5(4.1), p=0.04$ ] scores 